\title{
The orbital-motion-limited regime of cylindrical Langmuir probes
}

\author{
J. R. Sanmartín ${ }^{\text {a) }}$ \\ ETSI Aeronáuticos, Universidad Politécnica de Madrid 28040, Madrid, Spain \\ R. D. Estes \\ Harvard-Smithsonian Center for Astrophysics, 60 Garden St., Cambridge, Massachusetts 02138
}

(Received 29 June 1998; accepted 7 October 1998)

\begin{abstract}
An asymptotic analysis of electron collection at high bias $\Phi_{p}$ serves to determine the domain of validity of the orbital-motion-limited (OML) regime of cylindrical Langmuir probes, which is basic for the workings of conductive bare tethers. The radius of a wire collecting OML current in an unmagnetized plasma at rest cannot exceed a value, $R_{\max }$, which is found to exhibit a minimum as a function of $\Phi_{p}$; at $\Phi_{p}$ values of interest, $R_{\max }$ is already increasing and is larger than the electron Debye length $\lambda_{D e}$. The breakdown of the regime relates to conditions far from the probe, at electron energies comparable to the ion thermal energy, $k T_{i} ; R_{\max }$ is found to increase with $T_{i}$. It is also found that (1) the maximum width of a thin tape, if used instead of a wire, is $4 R_{\max }$; (2) the electron thermal gyroradius must be larger than both $R$ and $\lambda_{D e}$ for magnetic effects to be negligible; and (3) conditions applying to the tether case are such that trapped-orbit effects are negligible. (C) 1999 American Institute of Physics. [S1070-664X(99)03801-X]
\end{abstract}

\section{INTRODUCTION}

A space tether is a cable that connects orbiting end masses and is kept vertical by the gravity gradient force. If conductive, its electrodynamic interaction with the ionosphere and geomagnetic field has potential applications that range from power generation and propulsion ${ }^{1}$ to the use of wave $^{2,3}$ and particle emissions. ${ }^{4}$ The standard tether carries insulation, and has end devices to establish and control electrical contact with the ionospheric plasma. The bottleneck for such applications is the efficient capture of ionospheric electrons at the anodic end of the tether: the electron gyroradius and Debye length are so small compared to any useful, threedimensional, passive anode that both magnetic guiding and electric shielding greatly reduce collection. This has motivated work on active contactors that create a plasma cloud to bridge the ionosphere. 5,6

Using uninsulated (bare) tethers, whose anodic segment might itself capture electrons passively with no shielding or magnetic effects, has been proposed as a simple alternative. ${ }^{7}$ An anodic segment in the kilometer range leads to quite large (in addition to effective) collecting areas; also, bare tethers prove fairly insensitive to regular drops in plasma density along an orbit. ${ }^{8,9}$ A National Aeronautics and Space Administration (NASA) experiment (Propulsive Small Expendable Deployer System) will test a bare-tether collection in a 2000, Delta-2 rocket flight. The Marshall Space Flight Center is considering the use of bare tethers in the International Space Station, for reboost or secondary power generation. As a power generator, for instance, a bare tether would have a useful load and a cathodic contactor at its base, and electron collection, if optimal, would extend roughly to the upper $\left(m_{e} / m_{i}\right)^{1 / 5} \approx 1 / 7$ of the total length.

Note that each point of an electrodynamic bare tether

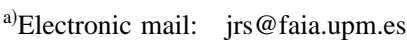

would collect current as if it were part of a cylinder uniformly polarized at the local tether bias. ${ }^{7}$ This is because of the enormous disparity between tether thickness and collecting length, which lie in the millimeter and kilometer ranges, respectively. Bare tether applications rest on the assumption that electron collection occurs in the optimal orbital-motionlimited (OML) regime of cylindrical probes. It is thus important to determine its parametric domain of validity.

Since OML current is proportional to the perimeter of the cross section, a large tether current may require a large perimeter. If the crosswise dimension is too large, however, the current will not reach the OML value because of electrical screening effects related to a comparatively short Debye length $\lambda_{D e}$. Here we determine the maximum radius of a cylinder collecting OML current in an unmagnetized plasma at rest, and how it depends on the ion temperature $T_{i}$ and the bias $\Phi_{p}$ (Secs. II and III). Values of the ratio $e \Phi_{p} / k T_{e}$ of interest for tethers $\left(T_{e} \sim 0.15 \mathrm{eV}, \Phi_{p} \sim 400 \mathrm{~V}\right)$ are $10^{2}$ times larger than values previously explored numerically.

We also determine what would be the maximum width of a thin tape, which has been proposed as a tether (Sec. IV). Next we consider how large the thermal electron gyroradius $l_{e}$ has to be for magnetic effects to be negligible (Sec. V); again, if the crosswise dimension is too large, the current would not reach the OML value because of magnetic guiding effects due to a short $l_{e}$. Results are discussed in Sec. VI.

\section{CIRCULAR CYLINDER AT REST IN AN UNMAGNETIZED PLASMA}

The electron current $I$ to a sufficiently long cylinder at rest in a collisionless, unmagnetized, Maxwellian plasma of density $N_{\infty}$ and temperatures $T_{e}$ and $T_{i}$ may be written in dimensionless form as $I=I_{t h}$ times a function of $R / \lambda_{D e}$, $e \Phi_{p} / k T_{e}, T_{i} / T_{e}$. Here, $I_{t h} \equiv 2 \pi R L e N_{\infty} \sqrt{k T_{e} / 2 \pi m_{e}}$ is the thermal or random current, $\lambda_{D e}$ is $\sqrt{k T_{e} / 4 \pi e^{2} N_{\infty}}$, and $R, L$, 


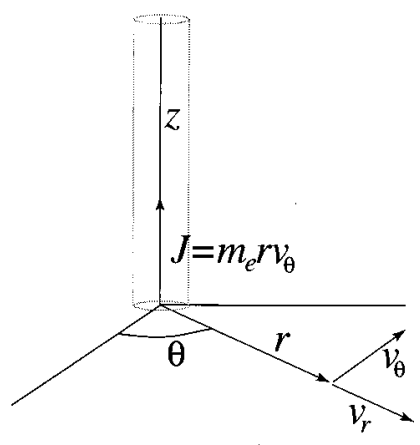

FIG. 1. Geometry of cylindrical probe and electron motion.

and $\Phi_{P}$ are the probe radius, length, and bias. In general, the determination of electron trajectories to obtain the current requires solving Poisson's equation for the potential $\Phi(r)$,

$$
\frac{\lambda_{D i}^{2}}{r} \frac{d}{d r}\left(r \frac{d}{d r} \frac{e \Phi}{k T_{i}}\right)=\frac{N_{e}}{N_{\infty}}-\frac{N_{i}}{N_{\infty}} \quad\left(\lambda_{D i}=\lambda_{D e} \sqrt{T_{i} / T_{e}}\right),
$$

with boundary conditions $\Phi=\Phi_{p}>0$ at $r=R, \Phi \rightarrow 0$ as $r$ $\rightarrow \infty$.

Both the electric field $-\nabla \Phi$ and the probe acting as a sink of particles affect the densities $N_{e}$ and $N_{i}$, and thus $\Phi(r)$ itself. The basic problem in probe theory usually lies in the attracted-particle density $N_{e}$. Actually, for the $e \Phi_{p}$ $\gg k T_{i}, k T_{e}$ values of interest here, the repelled-particle density $N_{i}$ is accurately given by the simple Boltzmann law,

$$
N_{i} \approx N_{\infty} \exp \left(-e \Phi / k T_{i}\right)
$$

except near the probe, where $N_{i}$ (as the ion current itself) is exponentially small anyway. Equation (2) makes convenient to normalize $\Phi$ and $r$ with the ion parameters $T_{i}$ and $\lambda_{D i}$.

Since all electrons originate at infinity, and the Vlasov equation conserves the distribution function $f(\bar{r}, \bar{v})$ along electron orbits, we have $f(\bar{r}, \bar{v})=f_{M}\left(v_{\infty}\right)$ (undisturbed Maxwellian) if the $\bar{r}, \bar{v}$ orbit, traced back in time, reaches infinity; otherwise, we have $f(\bar{r}, \bar{v})=0$. Note next that both axial velocity $v_{z}$, and transverse energy (Fig. 1),

$$
\frac{m_{e}}{2} v_{r}^{2}+\frac{m_{e}}{2} v_{\theta}^{2}-e \Phi \equiv E\left(=\frac{m_{e}}{2} v_{\infty}^{2}-\frac{m_{e}}{2} v_{z}^{2}\right),
$$

are also conserved along orbits; the values $\bar{r}, \bar{v}$ thus determine $v_{\infty}$, and, consequently, the $f_{M}$ value, in terms of the local potential $\Phi \equiv \Phi(r)$. The density $N_{e}$ at each particular radius $r$ may then be expressed as an integral of the undisturbed Maxwellian distribution function over appropriate velocity ranges. ${ }^{10} \mathrm{~A}$ trivial $v_{z}$ integration, and a change of variables $v_{r}, v_{\theta} \rightarrow E, J$, yields

$$
\frac{N_{e}}{N_{\infty}}=\iint \frac{\exp \left(-E / k T_{e}\right) d E d J}{2 \pi k T_{e} \sqrt{J_{r}^{2}(E)-J^{2}}},
$$

where we introduced the angular momentum $J \equiv m_{e} r v_{\theta}$, and defined

$$
J_{r}^{2}(E) \equiv 2 m_{e} r^{2}[E+e \Phi(r)] .
$$

The $E$-integral, which only covers positive values must be carried out once for $v_{r}<0$ (incoming electrons) and again for $v_{r}>0$ (electrons that have turned outwards at a radius between $r$ and $R$ ). The $J$-integral can be made to cover just positive values by writing $d J \rightarrow 2 d J$. The $E-J$ domain of integration in Eq. (4) is $r$-dependent because of both the electric field and the sink effect of the probe:

(i) For an incoming electron of energy $E>0$ to actually reach $r, v_{r^{\prime}}^{2}$ must have been positive throughout the entire range $r<r^{\prime}<\infty$. Using both $J$ and (5) in Eq. (3),

$$
m_{e}^{2} r^{2} v_{r}^{2}=J_{r}^{2}(E)-J^{2},
$$

and using now the fact that $J$ is also conserved, its range of integration at that energy will clearly be

$$
0<J<J_{r}^{*}(E) \equiv \operatorname{minimum}\left\{J_{r^{\prime}}(E) ; r \leqslant r^{\prime}<\infty\right\} .
$$

In general, the minimum occurs at a different $r^{\prime}$ for each energy $E$. If $J_{r}^{*}(E)$ differs from $J_{r}(E)$, those electrons in the range $J_{r}^{*}(E)<J<J_{r}(E)$, for which $v_{r}^{2}$ would actually be positive, never reach $r$, and must thus be excluded from the integral in (4); one says that there is an effective potential barrier for $r$, at energy $E$.

(ii) For an $E$-electron outgoing at $r$ the $J$-range of integration will be

$$
J_{R}^{*}(E)<J<J_{r}^{*}(E),
$$

electrons in the range $0<J<J_{R}^{*}(E)$ having disappeared at the probe.

Equation (4) may now be written as

$$
\begin{aligned}
\frac{N_{e}}{N_{\infty}}= & \int_{0}^{\infty} \frac{d E}{\pi k T_{e}} \exp \left(\frac{-E}{k T_{e}}\right) \\
& \times\left[2 \sin ^{-1} \frac{J_{r}^{*}(E)}{J_{r}(E)}-\sin ^{-1} \frac{J_{R}^{*}(E)}{J_{r}(E)}\right],
\end{aligned}
$$

half the first term in the bracket being the $\nu_{r}<0$ contribution. The current itself is easily found to be

$$
I=2 R L e N_{\infty} \sqrt{\frac{2 e \Phi_{P}}{m_{e}}} \times \int_{0}^{\infty} \frac{d E}{k T_{e}} \exp \left(\frac{-E}{k T_{e}}\right) \frac{J_{R}^{*}(E)}{J_{R}(0)} .
$$

We note at this point that, through its dependence on $J_{r}^{*}(E)$ [and $J_{R}^{*}(E)$ ], the density $N_{e}$ is a functional of $\Phi(r)$ and thus cannot be known, for use in solving Eq. (1) for $\Phi(r)$, before the potential itself is found; this results in a complex, iterative numerical solution of Poisson's equation. ${ }^{11}$

A hypothetical potential with no barriers at all $\left[J_{r}^{*}(E)\right.$ $=J_{r}(E)$ for $\left.0 \leqslant E<\infty, R \leqslant r<\infty\right]$ would everywhere reduce $N_{e}$ in (7) to a function of the local radius and potential,

$$
\frac{N_{e}}{N_{\infty}}=1-\int_{0}^{\infty} \frac{d E}{\pi k T_{e}} \exp \left(\frac{-E}{k T_{e}}\right) \sin ^{-1} \frac{J_{R}(E)}{J_{r}(E)},
$$

with $J_{r}(E)$ given in Eq. (5). This would allow a ready solution of Eq. (1). As we shall see, however, Eq. (9) does not fully apply to the case of interest here, which corresponds to the maximum possible current in (8). Since we have $J_{R}^{*}(E)$ $\leqslant J_{R}(E)$ from the definition of $J_{r}^{*}(E)$ in (6), current is maximum under the condition $J_{R}^{*}(E)=J_{R}(E)$, for $0<E<\infty$ [no potential barrier for just radius $R$; note that the second term 


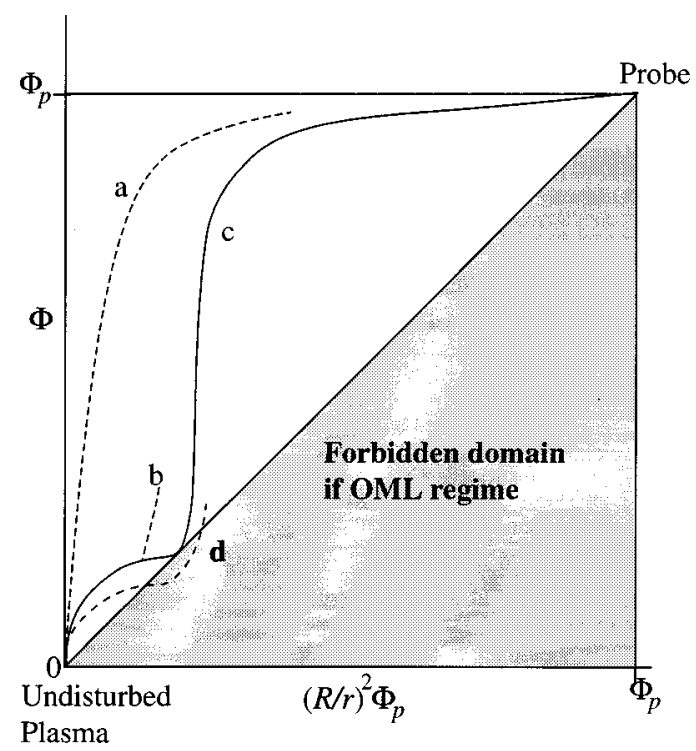

FIG. 2. Schematics of potential $\Phi$ versus $\Phi_{P} R^{2} / r^{2}$ for profiles $a$ and $b(R$ $\left.<R_{\max }\right), c\left(R=R_{\max }\right)$, and $d\left(R>R_{\max }\right)$, with $R_{\max }$ the largest radius for the OML regime to hold. The hypothetical profile $a$ would have no potential barriers.

in the bracket of (7) does reduce to a function of the local $r$ and $\Phi]$. This is the OML current; with $E \sim k T_{e} \ll e \Phi_{p}$, we have $J_{R}(E) \approx J_{R}(0)$, Eq. (8) giving

$$
\begin{aligned}
I_{\mathrm{OML}} & \approx 2 R L N_{\infty} e \sqrt{2 e \Phi_{P} / m_{e}} \\
& =I_{t h} \times \sqrt{4 e \Phi_{P} / \pi k T_{e}} \quad\left(e \Phi_{p} \gg k T_{e}\right) .
\end{aligned}
$$

With the current known, however, there would be no need for solving Eq. (1), except for the very purpose of the present work: determining the parametric domain for the OML regime to hold. For $e \Phi_{p} \gg k T_{e}, k T_{i}$, this problem comes out to be reasonably simple.

Note that to have $J_{r}^{*}(E)=J_{r}(E)$ for the entire range 0 $\leqslant E<\infty$ at a particular $r$, it suffices to have $J_{r}^{*}(0)=J_{r}(0)$ [for $E$ large enough one has $J_{r}^{2}(E) \approx 2 m_{e} r^{2} E$ and thus $\left.J_{r}^{*}(\infty) / J_{r}(\infty)=1\right]$. From $J_{r}^{2}(0) \propto r^{2} \Phi(r)$ it follows that the condition of no potential barrier for a radius $r$ is

$$
r^{2} \Phi(r) \leqslant r^{\prime 2} \Phi\left(r^{\prime}\right) \quad\left(r \leqslant r^{\prime}<\infty\right) .
$$

In particular, the OML condition, $J_{R}^{*}(E)=J_{R}(E)$ for $0<E$ $<\infty$, requires the potential to satisfy

$$
R^{2} \Phi_{p} \leqslant r^{2} \Phi(r) \quad(R \leqslant r<\infty)
$$

Condition (11) can be conveniently illustrated by displaying $\Phi$ versus $\Phi_{p} R^{2} / r^{2}$ for potential profiles (Fig. 2); (11) shows that the profile for $R=R_{\max }$ (the maximum radius for the OML regime to hold, with other parameters fixed) would just touch the diagonal in the figure, as in the case of profile $c$. Profiles $a$ and $b$ would lie in the OML regime, whereas $d$ would not.

Finally, note that the extreme condition $J_{r}^{*}(E)=J_{r}(E)$ for $0<E<\infty, R \leqslant r<\infty$, which led to Eq. (9), would require the potential to satisfy

$$
d\left(r^{2} \Phi\right) / d r \geqslant 0 \quad(R<r<\infty) .
$$

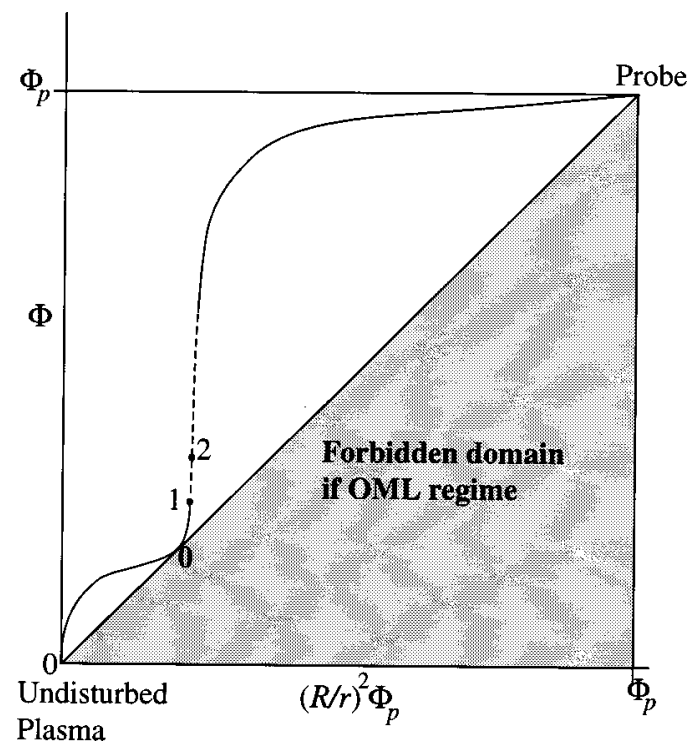

FIG. 3. Potential profile for $R=R_{\max }$. The plasma is quasineutral below point 1 ; below point 0 there are no potential barriers. The broad, ion-free region above the thin layers at points 1 and 2 is free of space-charge effects near the probe.

Condition (12), which is, of course, more restrictive than (11), requires the ordinate-to-abscissa profile ratio in Fig. 2, $\Phi /\left(\Phi_{p} R^{2} / r^{2}\right)$, to monotonically decrease throughout when moving to the right in the figure; only the hypothetical case $a$ satisfies (12). Note, however, that if $d\left(r^{2} \Phi\right) / d r$ is positive just beyond some radius $r_{0}$, then we do have

$$
J_{r}^{*}(E)=J_{r}(E), \quad \text { for } 0<E<\infty, \quad r_{0} \leqslant r<\infty,
$$

and Eq. (7) reduces to (9) for $r>r_{0}$. Cases $b-d$ present this property; $r_{0}$ would be the radius where the profile ratio $\Phi /\left(\Phi_{P} R^{2} / r^{2}\right)$ is minimum. For case $c$, which is reproduced in Fig. 3, the corresponding profile point lies on the diagonal.

\section{MAXIMUM RADIUS FOR OML VALIDITY}

Figure 3 corresponds to the actual profile for $R=R_{\max }$, at large $e \Phi_{p} / k T_{i}$; this may be taken as an ansatz that is used in solving Poisson's equation and verified in the solution. We carry out an asymptotic analysis of Eq. (1) for $e \Phi_{p} / k T_{i} \gg 1$, following closely a classical study, which, however, was developed for a monoenergetic attractedparticle distribution function, and for the non-OML, small $\lambda_{D i} / R$, regime. ${ }^{12}$ The profile presents several distinct regions.

(1) Both the quasineutral approximation, $N_{e} \approx N_{i}$, and the no barrier condition (13) hold below point 0 in Fig. 3. Condition (13) may be illustrated by considering the $r$-family of straight lines $J^{2}=J_{r}^{2}(E)$ of the $E-J^{2}$ plane in the range $r \geqslant r_{0}$ : for $r$ increasing, the corresponding line keeps moving to the right for all positive energies (Fig. 4a). The use of (2) and (9) in $N_{e} \approx N_{i}$ determines $\Phi(r)$; for $r$ large enough, one has $\Phi \propto 1 / r$, as suggested in Fig. 3. Values $\Phi_{0}$ and $r_{0}$ for point 0 , lying on the diagonal, are then given exactly by 

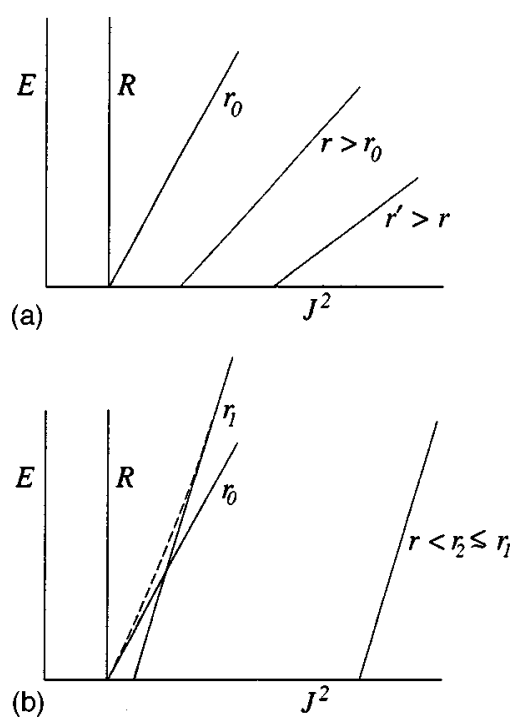

FIG. 4. (a) Straight lines of the $r$-family $J^{2}=J_{r}^{2}(E)\left[J_{r}^{2}(E)\right.$ defined in Eq. (5)], for the probe, point 0 in Fig. 3, and any two radii $r^{\prime}>r$ beyond $r_{0}$. (b) Envelope $J_{\text {env }}^{2}(E)$ (dashed curve) for $r$-family lines $J^{2}=J_{r}^{2}(E)$ in the range $r_{1}<r<r_{0}$, and limit lines for points 0 and 1 ; the envelope is approximately described by Eq. (16). At the top of the thin layers, and for most of the broad region above, in Fig. 3, $r$-lines lie far to the right, as indicated; as the probe is approached, however, the $r$-line would move back to the left, finally reaching the probe line.

$\left[1+\exp \left(e \Phi_{0} / k T_{e}\right) \operatorname{erfc}\left(\sqrt{e \Phi_{0} / k T_{e}}\right)\right] \exp \left(e \Phi_{0} / k T_{i}\right)=2$,

$\Phi_{0}=\Phi_{P} R^{2} / r_{0}^{2}$,

(2) The quasineutral approximation remains valid from point 0 up to a point 1 where $-d \Phi / d r \rightarrow \infty$ (Fig. 3). Now, however, there is an $r$-dependent energy range with potential barrier. Since we have $r_{1}<r_{0}$ and $r_{1}^{2} \Phi_{1}>r_{0}^{2} \Phi_{0}$, the $r$-lines for points 0 and 1 must meet at some positive energy, as shown in Fig. 4b. Also shown is the envelope $J^{2}=J_{\text {env }}^{2}(E)$ of the set of $r$-lines between points 0 and 1 , which is determined by the equations $J^{2}-J_{r}^{2}(E)=0, \partial\left[J^{2}-J_{r}^{2}(E)\right] / \partial r$ $=0$, leading to the parametric representation

$$
\begin{aligned}
& J^{2}=J_{e n v}^{2}(r) \equiv-m_{e} r^{3} e d \Phi / d r, \\
& E=E_{e n v}(r) \equiv-e \Phi(r)-1 / 2 r e d \Phi / d r .
\end{aligned}
$$

The envelope is tangent to each $r$-line at the $E, J^{2}$ point given by Eqs. (15a), (15b). Since $E$ and $J^{2}$ diverge with $-d \Phi / d r$ in (15a), (15b), as $r \rightarrow r_{1}$, the envelope is asymptotic to the $r_{1}$-line. Also, it is tangent to the $r_{0}$-line at $E=0$ (Fig. 4b); condition $E_{\text {env }}(r)=0$ in (15b) corresponds to a minimum of $r^{2} \Phi$, the profile becoming tangent to the diagonal in Fig. 3 when approaching point 0 from above. [The quasineutral solution below 0 has no such property, thus breaking down at that point; however, using locally the full equation (1), together with (2) and (9), suffices to round the profile at 0 , with no effect beyond its immediate neighborhood.] A simple but accurate approximation for $J_{e n v}(E)$ can now be readily obtained from Fig. $4 \mathrm{~b}$, using the $r_{0^{-}}$and $r_{1}$-lines without the knowledge of $\Phi(r)$,

$$
J_{e n v}^{2}(E)=J_{r_{1}}^{2}(E)-\frac{2 m_{e}\left(r_{1}^{2} e \Phi_{1}-r_{0}^{2} e \Phi_{0}\right)^{2}}{r_{1}^{2} e \Phi_{1}-r_{0}^{2} e \Phi_{0}+\left(r_{0}^{2}-r_{1}^{2}\right) E} .
$$

The values $r_{0}$ and $\Phi_{0}$ are taken from (14a), (14b) but $r_{1}$ and $\Phi_{1}$ are yet unknown.

For any radius $r$ between $r_{1}$ and $r_{0}$ we would now have

$$
\begin{aligned}
J_{r}^{*}(E) & =J_{e n v}(E), \text { for } E<E_{\text {env }}(r), \\
& =J_{r}(E), \text { for } E>E_{\text {env }}(r) .
\end{aligned}
$$

As $r$ approaches $r_{1}$, one has $E_{\text {env }}(r) \rightarrow \infty$ in (15b); Eq. (17a) for $J_{r}^{*}(E)$ may then be used in Eq. (7) for $N_{e}$ throughout the entire range $0<E<\infty$. Near $r_{1}$ the quasineutrality equation giving $\Phi(r)$ then reads as

$$
\begin{aligned}
\frac{N_{e}}{N_{\infty}} \equiv & \int_{0}^{\infty} \frac{d E}{\pi k T_{e}} \exp \left(\frac{-E}{k T_{e}}\right) \\
& \times\left[2 \sin ^{-1} \frac{J_{e n v}(E)}{J_{r}(E)}-\sin ^{-1} \frac{J_{R}(0)}{J_{r}(E)}\right] \\
= & \exp \left(\frac{-e \Phi(r)}{k T_{i}}\right) .
\end{aligned}
$$

Note that neither $J_{e n v}(E)$ nor $J_{R}(0)$ involve the local values of $r$ or $\Phi$. The derivative of (18) with respect to $\Phi$ at $r_{1}$, where $d r / d \Phi$ vanishes, then reads as

$$
\begin{aligned}
\int_{0}^{\infty} & \frac{d E \exp \left(-E / k T_{e}\right)}{2 \pi\left(E+e \Phi_{1}\right) T_{e} / T_{i}} \\
& \times\left[2 \sqrt{\frac{J_{e n v}^{2}(E)}{J_{r_{1}}^{2}(E)-J_{e n v}^{2}(E)}}-\sqrt{\frac{J_{R}^{2}(0)}{J_{r_{1}}^{2}(E)-J_{R}^{2}(0)}}\right] \\
& =\exp \left(\frac{-e \Phi_{1}}{k T_{i}}\right) .
\end{aligned}
$$

Using (16) in Eqs. (18), evaluated at $r_{1}$, and (19) yields $r_{1}$ and $\Phi_{1}$, thus fully determining $J_{e n v}(E)$. Figure 5a shows $e \Phi_{0} / k T_{i}\left(=e \Phi_{P} R^{2} / k T_{i} r_{0}^{2}\right), e \Phi_{1} / k T_{i}$, and $\sigma_{1} \equiv e \Phi_{P} R^{2} /$ $k T_{i} r_{1}^{2}$ as functions of $T_{e} / T_{i}$.

(3) Above point 1 in Fig. 3 there are two thin, nonquasineutral layers that take the solution to a radius $r_{2}$ a bit closer to the probe, and to values $\Phi$ satisfying $\Phi_{1} \ll \Phi$ $\ll \Phi_{p}$, with the $r$-line moving far to the right in Fig. 4b. Note that both $e \Phi_{0}$ and $e \Phi_{1}$ are of the order of $k T_{i}$ whereas $e \Phi_{p} / k T_{i}$ is very large $\left(\sim 10^{3}, 10^{4}\right)$. If Fig. 3 were drawn to scale, the near-vertical potential drop in the two thin layers, down to point 1 , would occur very close to the $\Phi$-axis, and point 0 would lie very close to the origin.

The structures of the two layers are considered in Appendix A. At the top of the second layer, $\Phi$ is found to increase in Fig. 3 as $\Phi \propto\left(r_{2}-r\right)^{4 / 3}$. The difference $r_{1}-r_{2}$ vanishes with $k T_{i} / e \Phi_{P}$, and is small for the high $\Phi_{P}$ values of interest. Defining $\sigma_{2} \equiv e \Phi_{P} R^{2} / k T_{i} r_{2}^{2}$ we get, from Eq. (A6) in Appendix A,

$$
\begin{aligned}
& \sigma_{2}=\sigma_{1}\left[1+2 \xi_{2}\left(\frac{2 \sigma_{1}^{2}}{\lambda \mu}\right)^{1 / 5}\left(\frac{k T_{i}}{e \Phi_{P}}\right)^{2 / 5}\left(\frac{\lambda_{D i}}{R}\right)^{4 / 5}\right], \\
& \xi_{2} \approx 3.45,
\end{aligned}
$$



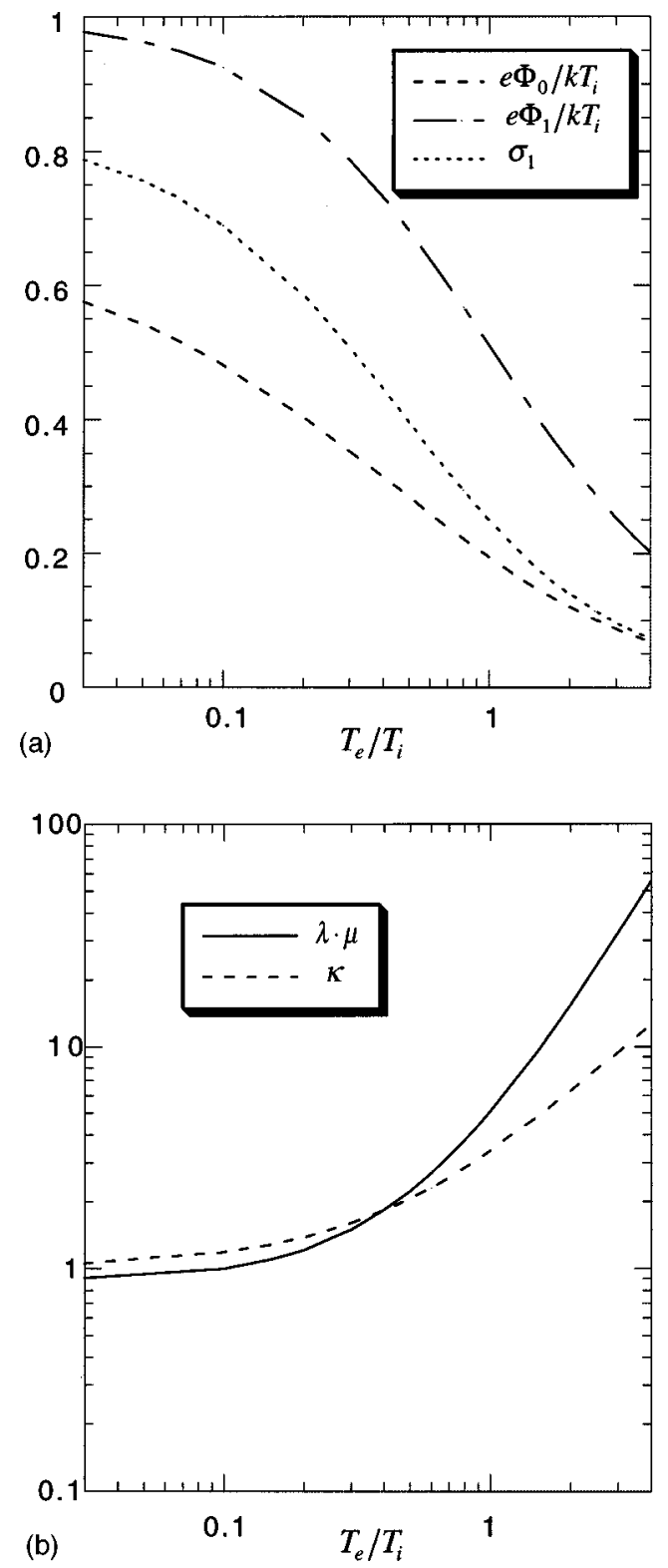

FIG. 5. (a) Dimensionless quantities $e \Phi_{0} / k T_{i}\left(=e \Phi_{P} R^{2} / k T_{i} r_{0}^{2}\right)$, $e \Phi_{1} / k T_{i}$, and $e \Phi_{P} R^{2} / k T_{i} r_{1}^{2} \equiv \sigma_{1}$, versus $T_{e} / T_{i}$. (b) Dimensionless quantities $\kappa$ [defined in Eq. (21)] and $\lambda \mu(\lambda$ and $\mu$ defined in Appendix A) versus $T_{e} / T_{i}$.

with $\lambda \mu$ given in Fig. $5 b$.

(4) In the broad region between the second thin layer and the probe, we have $e \Phi / k T_{i} \sim \Phi / \Phi_{1}$ large (Fig. 3); $N_{i} / N_{\infty}$ is then exponentially small and may be neglected in Poisson's equation. Also, $r$-lines lie far to the right in Fig. 4b; we then have both $J_{r}^{*}(E)=J_{\text {env }}(E)$, and $J_{\text {env }}(E) \sim J_{R}(0) \ll J_{r}(E)$ $\approx J_{r}(0)$. We may thus simplify the expression for $N_{e} / N_{\infty}$ in Eq. (18) and use it in (1), which takes the form

$$
\frac{\lambda_{D i}^{2}}{r} \frac{d}{d r}\left(r \frac{d}{d r} \frac{e \Phi}{k T_{i}}\right) \approx \frac{N_{e}}{N_{\infty}} \approx \frac{\kappa}{\pi} \frac{R}{r} \sqrt{\frac{\Phi_{P}}{\Phi}},
$$

where

$$
\kappa \equiv 2 \int_{0}^{\infty} \frac{d E}{k T_{e}} \exp \left(\frac{-E}{k T_{e}}\right) \frac{J_{e n v}(E)}{J_{R}(0)}-1
$$

is a function of $T_{e} / T_{i}$, given in Fig. $5 \mathrm{~b}$ too. Introducing ${ }^{12}$

$$
\begin{aligned}
u & \equiv \ln \frac{r_{2}}{r}, \\
g & \equiv\left[\pi \frac{\sqrt{\sigma_{2}}}{\kappa} \frac{\lambda_{D i}^{2}}{R^{2}} \frac{k T_{i}}{e \Phi_{P}}\right]^{2 / 3} \frac{e \Phi}{k T_{i}},
\end{aligned}
$$

Eq. (20) becomes

$$
d^{2} g / d u^{2}=e^{-u} / \sqrt{g} .
$$

Matching to the top of the second thin layer yields the boundary conditions

$$
\begin{aligned}
& g=0, \quad d g / d u=0 \quad\left(g \sim u^{4 / 3} \text { as } u \rightarrow 0\right), \\
& \text { at } u=0 \quad\left(r=r_{2}\right) .
\end{aligned}
$$

This fully determines $g(u)$, which is a parameter-free function. Finally, once $g(u)$ is found numerically, setting $\Phi$ $=\Phi_{p}$ at $r=R \equiv R_{\max }$ in (22a), (22b) yields a relation between parameters, i.e., determines $R_{\max }$,

$$
\frac{R_{\max }}{\lambda_{D i}}=\frac{\sqrt{\pi}\left(\sigma_{2} e \Phi_{P} / k T_{i}\right)^{1 / 4}}{\sqrt{\kappa}\left[g\left(\ln \sqrt{e \Phi_{p} / k T_{i} \sigma_{2}}\right)\right]^{3 / 4}},
$$

with $\sigma_{2}$ given by Eq. (A6), where $R=R_{\max }$, and $\sigma_{1}\left(T_{e} / T_{i}\right)$, and $\lambda \mu\left(T_{e} / T_{i}\right)$ and $\kappa\left(T_{e} / T_{i}\right)$ given in Figs. 5a and 5b, respectively. Since $\sqrt{e \Phi_{P} / k T_{i} \sigma_{2}} \equiv r_{2} / R$ is large, one might use both $\sigma_{2} \approx \sigma_{1}$ and the asymptotic form of $g(u)$ at large $u$,

$$
g \approx c \cdot(u-b), \quad c \approx 2.0854, \quad b \approx 0.3511,
$$

to get a simpler, approximate law for $R_{\max }$,

$$
\frac{R_{\max }}{\lambda_{D i}} \approx \frac{\sqrt{\pi}\left(\sigma_{1} e \Phi_{P} / k T_{i}\right)^{1 / 4}}{\sqrt{\kappa} c^{3 / 4}\left(\ln \sqrt{e \Phi_{P} / k T_{i} \sigma_{1}}-b\right)^{3 / 4}} .
$$

The behavior $g \approx c \cdot(u-b)$ shows that the high bias makes space-charge effects negligible within some neighborhood of the probe (even though $R$ is not small compared with $\left.\lambda_{D e}, \lambda_{D i}\right)$. Using Eqs. (22a), (22b), $\Phi(r)$ takes the form of a (logarithmic) solution to the two-dimensional (2D) Laplaceequation, with $N_{e}-N_{i}$ thus ignored in Eq. (1),

$$
g \approx c \cdot(u-b) \rightarrow \Phi \approx \Phi_{P}\left[1-\frac{1}{\ln \sqrt{e \Phi_{P} / k T_{i} \sigma_{1}}-b} \ln \frac{r}{R}\right] .
$$

This is fortunate because the approximation for $N_{e} / N_{\infty}$ in Eq. (20) would actually fail near the probe: as one approaches it, moving toward the upper right corner in Fig. 3, the $r$-line moves back to the left in Fig. 4b, to finally reach the $R$-line at the probe.

Figure 6 shows $R_{\max } / \lambda_{D i}$ versus $e \Phi_{p} / k T_{i}$ for different values of $T_{e} / T_{i}$, using (24a). The dependence on the repelled particle temperature $T_{i}$ is clearly a consequence of $e \Phi_{0}$ and $e \Phi_{1}$ being of order of $k T_{i}$; it is also related to the well-known fact that the current to a probe, in the opposite, thin sheath limit, depends on the repelled particle temperature. Note that $R_{\max }$ goes through a minimum as the bias $\Phi_{p}$ increases, and exceeds $\lambda_{D i}$ at high enough $\Phi_{p}$. Numerical calculations for the range $e \Phi_{p} / k T_{i}<25$ had shown $R_{\max }$ decreasing monotonically with increasing bias, ${ }^{11}$ our results are 


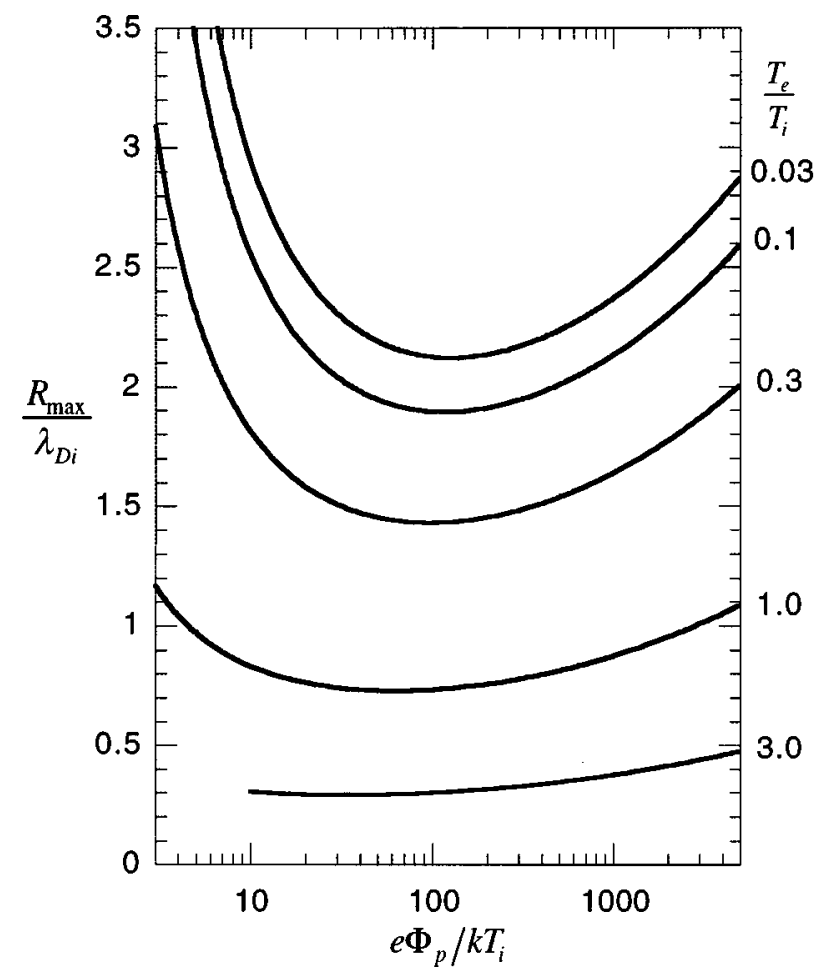

FIG. 6. $R_{\max } / \lambda_{D i}$ versus $e \Phi_{P} / k T_{i}$ for several values of temperature ratio $T_{e} / T_{i}$, using Eq. (24a).

in reasonable agreement with those calculations for moderate $e \Phi_{p} / k T_{i}$, although our analysis strictly is only valid for very high bias. Using $g(u)$ and the approximation $\sigma_{2}=\sigma_{1}$, Eq. (24a) with $T_{e} / T_{i}$ fixed yields readily a minimum for $R_{\text {max }}$ at $u_{P} \equiv \ln \sqrt{e \Phi_{p} / k T_{i} \sigma_{1}} \cong 1.76$, or

$e \Phi_{P} / k T_{i}$ at minimum $=\exp (2 \times 1.76) \times \sigma_{1}\left(T_{i} / T_{e}\right) ;$

a comparison to Fig. 6 proves that Eq. (A6) has an important effect at moderate bias. Figure 7 shows $R_{\max } / \lambda_{D e}$ versus $e \Phi_{p} / k T_{e}$.

To understand how the minimum of $R_{\max }$ arises in Fig. 6 , note that the broad region described by (20) can be characterized by the values $r_{m}, \Phi_{m}$ of the profile point in Fig. 3 with tangent through the origin; this corresponds to maximum $r^{2} \Phi(r)$, i.e., maximum $\exp (-2 u) g(u)$ [and to minimum $N_{e} / N_{\infty}$ in Eq. (20)]. With $u_{m} \cong 0.63, g\left(u_{m}\right) \cong 0.86$ of order unity, we have

$$
r \sim r_{m} \sim r_{1}, \quad \Delta r \sim r_{1}-r_{m} \sim r_{1}, \quad \Phi \sim \Phi_{m} \sim \Phi_{P} / g\left(u_{P}\right),
$$

leading to characteristic values for both sides of (20),

$$
\frac{\lambda_{D i}^{2}}{R^{2} g\left(u_{P}\right)} \sim \sqrt{\frac{k T_{i}}{e \Phi_{P}}} \sqrt{g\left(u_{P}\right)}\left(\sim \frac{N_{e}}{N_{\infty}}\right),
$$

when $r_{1} \sim r_{0} \sim R \sqrt{e \Phi_{P} / k T_{i}}$ is used; relation (20') mirrors Eq. (24a). Note that increasing $\Phi_{P}$ would reduce the characteristic electron density (i.e., the space-charge that keeps $R_{\max }$ low) through the factor $\sqrt{k T_{i} / e \Phi_{P}}$ on the right-handside of $\left(20^{\prime}\right)$, but it would work the opposite way through $g\left(u_{P}\right)$ on both sides of it.

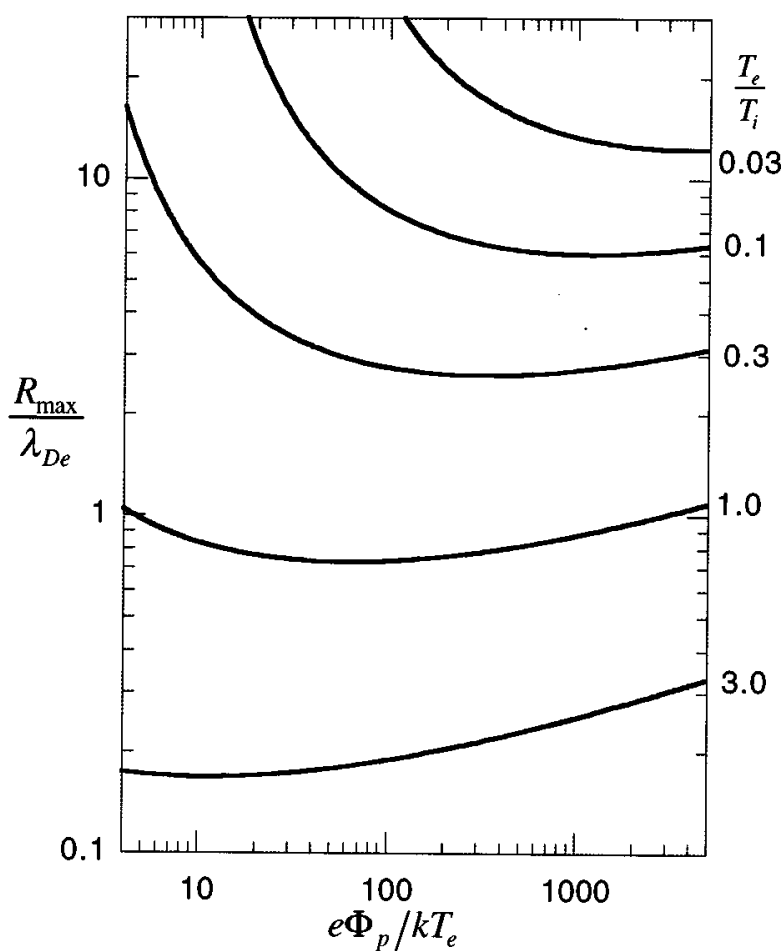

FIG. 7. The same as Fig. 6 , for $R_{\max } / \lambda_{D e}$ versus $e \Phi_{P} / k T_{e}$.

We have neglected collisions throughout. Collisions, however, even if unfrequent, might trap electrons in bounded orbits, thus creating additional space-charge that could affect $\Phi(r)$ and $I$. In Appendix B we establish under what conditions this effect should be negligible, and show that these conditions are satisfied in the tether case.

\section{THIN TAPE AT REST IN AN UNMAGNETIZED PLASMA}

In the OML regime, the current to a cylindrical probe has the important property of being independent of the shape of the cross section; it just depends on its perimeter, being given, in general, by Eq. (10) with $R$ replaced by perimeter $2 \pi$ (see Appendix C). ${ }^{13}$ The limits of OML validity, however, must be determined anew for every cross section. For a thin tape, angular momentum $J$ is not conserved, and there is no close-form expression such as (7) for $N_{e}$ [even though Eq. (4) would remain valid]. Nonetheless, we find that the high bias condition $\left(e \Phi_{p} \gg k T_{i}\right)$ makes it possible to approximately reduce the problem to the case of the circular cylinder.

Here it is convenient to use elliptical coordinates $v$ and $w$ defined by (Fig. 8)

$$
\begin{aligned}
x= & a \cos v \cosh w, \quad y=a \sin v \sinh w, \\
& (0 \leqslant v<2 \pi, \quad 0 \leqslant w<\infty) .
\end{aligned}
$$

Poisson's equation then reads as 


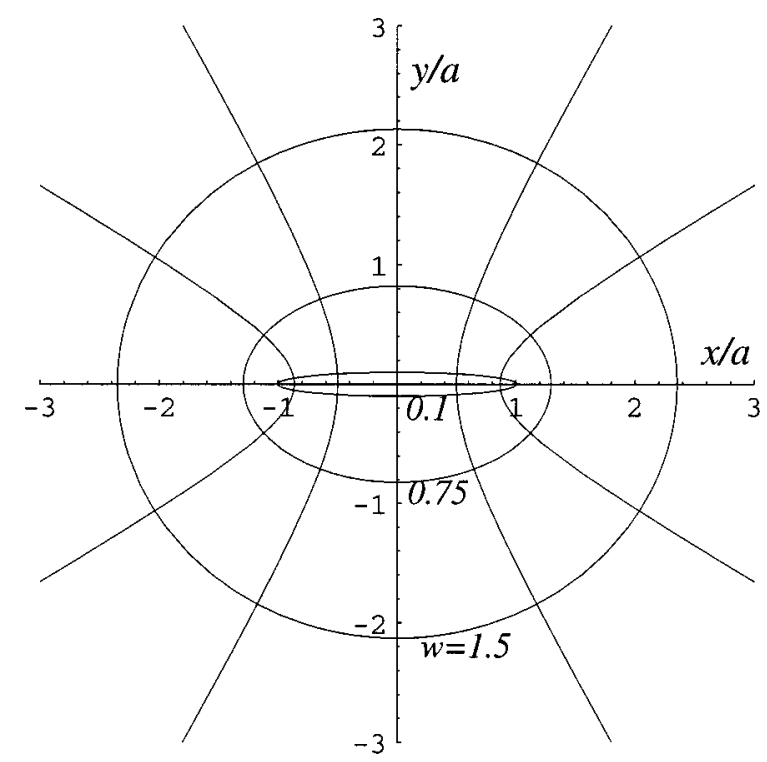

FIG. 8. Coordinate ellipses $w(x, y)=$ const for the orthogonal coordinates introduced in Sec. IV; they approach near-circles as $w$ increases. The limit ellipse $w=0(y=0,-a<x<a)$ represents the cross section of a thin tape of width $2 a$.

$$
\begin{gathered}
\frac{\lambda_{D i}^{2}}{a^{2}\left(\sinh ^{2} w+\sin ^{2} v\right)}\left(\frac{\partial^{2}}{\partial w^{2}}+\frac{\partial^{2}}{\partial v^{2}}\right) \frac{e \Phi}{k T_{i}} \\
=\frac{N_{e}}{N_{\infty}}-\exp \left(\frac{-e \Phi}{k T_{i}}\right) .
\end{gathered}
$$

Note that the cofocal coordinate ellipses $w(x, y)=$ const approach circles as $w$ increases; at large radial distances one has

$$
w=\ln \frac{r}{a}+\ln 2-\frac{x^{2}-y^{2}}{x^{2}+y^{2}} \frac{a^{2}}{4 r^{2}}+\cdots .
$$

One may reasonably use the approximation $w=\ln (2 r / a)$ for $w>w^{*}$, with $w^{*}=1.5$, say. Note also that the limit ellipse $w=0$ in Fig. 8 is the segment $y=0,-a<x<a$, which represents the cross section of a tape of width $2 a$ and negligible thickness.

Here, as in the case of a wire in Sec. III, the spacecharge will be ignorable within some neighborhood of the probe, which, for $e \Phi_{P} / k T_{i}$ large enough, extends into the region where $w$-ellipses are near-circles, that is, beyond $w$ $=w^{*}$. We may then argue that the potential $\Phi$ will be nearly independent of $v$ everywhere, i.e., $\Phi(w, v) \approx \Phi(w)$ (although the electric field will be radial for $w>w^{*}$ only). First, boundary conditions refer to just $w\left(\Phi=\Phi_{p}\right.$ at $w=0$ and $\Phi \rightarrow 0$ as $w \rightarrow \infty)$. Next, Poisson's equation, as given by (27), becomes

$$
\left(\frac{\partial^{2}}{\partial^{2} w}+\frac{\partial^{2}}{\partial^{2} v}\right) \frac{e \Phi}{k T_{i}} \approx 0
$$

for $w<w^{*}$, where the space charge may be neglected, and

$$
\frac{\lambda_{D i}^{2}}{a^{2} \sinh ^{2} w}\left(\frac{\partial^{2}}{\partial^{2} w}+\frac{\partial^{2}}{\partial^{2} v}\right) \frac{e \Phi}{k T_{i}} \approx \frac{N_{e}}{N_{\infty}}-\exp \left(\frac{-e \Phi}{k T_{i}}\right),
$$

for $w>w^{*}\left(e^{2 w}>e^{2 w^{*}} \gg 1\right)$, with some overlapping range of validity. In neither (28a) nor (28b) does $v$ show up explicitly.

Finally, with the ansatz $\Phi(w, v) \approx \Phi(w)$, the electron density in Eq. (28b) for $w>w^{*}$ would itself be a function of just $w, N_{e}=N_{e}(w)$. This is because, at a point in that region, incoming electrons, and outgoing electrons that did not reach values $w<w^{*}$, would find a radial field throughout their motion and conserve the angular momentum $J$; their contribution to $N_{e}$ would be a function of $r$, and thus, of $w$. Those outgoing electrons that had reached values $w<w^{*}$ and missed the probe, would have $J$ changed by a quantity $\Delta J$ that is easily shown to be small, $\Delta J \sim J / \ln \left(e \Phi_{p} / k T_{i}\right)$, as a result of the shallow (logarithmic) character of the potential in the vicinity of the probe, where the field is not radial; their contribution to $N_{e}$ would be weakly dependent on $v$. On the whole, we would thus have $N_{e} \approx N_{e}(w)$, consistently with the ansatz $\Phi(w, v) \approx \Phi(w)$.

With $\Phi=\Phi(w)$, and $w \approx \ln (2 r / a)$ for $w>w^{*}$, use of the probe boundary condition in Eq. (28a), which is also valid for some range beyond $w^{*}$, yields

$$
\begin{aligned}
\Phi & =\Phi_{p}[1-\alpha w], \quad \alpha=\text { const, } \\
& \rightarrow \Phi \approx \Phi_{p}\left[1-\alpha \ln \left(\frac{r}{a / 2}\right)\right], \quad \text { for } w>w^{*} .
\end{aligned}
$$

In addition, Eq. (28b) for $w>w^{*}$ may be shown to be exactly equivalent to (1), whose solution, as in Fig. 3 of Sec. III, will have an outer quasineutral region, two thin layers, and a broad, ion-free, inner region. Now this solution, rather than satisfying the boundary condition at the probe, must match smoothly the behavior given in (30), within the overlapping range of validity. Comparing Eqs. (25) and (30) shows that, beyond $w^{*}$, the solution behaves as in the case of a circular cylinder with an effective radius $R=a / 2$, the coefficient $\alpha$ being as given in (25).

This suggests that, with all other parameters given, the maximum width of a thin tape in the OML regime relates quite simply to the maximum radius of a circular cylinder,

$$
2 a_{\max }=4 R_{\max } \text {. }
$$

Results for a wire in Figs. 6 and 7 are therefore applicable here. Note that, although a tape allows twice as large a crosswise length as a wire, OML current is proportional to the perimeter, and thus a tape would only increase the maximum current by a factor $4 / \pi$, or 27 percent. A tape may be preferable, however, for other reasons: a cylinder with $R_{\max }$ might be too heavy and rigid; ${ }^{4}$ a tape may lead to a shorter tether. ${ }^{8,9}$

One must still take into account the fact that the Laplace potential (29), for the region $w<w^{*}$, is quite different from the potential (25). It then comes out that a tape, contrary to a circular cylinder, never collects the full OML current, although this has no practical consequences. There are potential barriers in the vicinity of any flat collecting surface, the effects being weak, however, in the case of a shallow 2D Laplace potential. ${ }^{13}$ Using (29) we find that potential barriers around the tape lie in a thin region of thickness $\sim a / \ln \left(e \Phi_{p} / k T_{i}\right)$ and that current reduction below the OML value is of order $\left[1 / \ln \left(e \Phi_{p} / k T_{i}\right)\right]^{2}$, or about 1 percent; we 
give details of these calculations in Appendix C. Equation (31) should then properly read that current to a tape keeps very close to the OML value for $a \leqslant 2 R_{\max }$.

\section{CIRCULAR CYLINDER AT REST IN A MAGNETIZED PLASMA}

As in the previous section, there is no closed-form expression for $N_{e}$ in the presence of an uniform magnetic field B, which allows for only two constants of the motion: energy and canonical angular momentum. Overall use of these two constants leads to the Parker-Murphy current law, which takes the character of an upper bound at the high bias of interest. ${ }^{14}$ For $e \Phi_{p} \gg k T_{e}$ and cylindrical geometry one has

$$
I_{\mathrm{PM}} \approx I_{\mathrm{OML}} \sqrt{\pi / 2} \times l_{e} / R,
$$

where $l_{e}$ is the electron thermal gyroradius,

$$
l_{e} \equiv \sqrt{k T_{e} / m_{e}} / \Omega_{e} \propto 1 / B \quad\left(\Omega_{e} \equiv e B / m_{e}\right) .
$$

Equation (32) suggests that if $l_{e} / R$ is large, $I_{\mathrm{OML}}$ then lying well below the $I_{\mathrm{PM}}$ bound, the OML current should hardly be affected by magnetic effects.

To get more definite results, consider electron motion in the presence of the electric field due to probe and plasma, $-\nabla \Phi(x, y)$ (probe and $z$ axes coincident as in Fig. 1), and a uniform magnetic field $\mathbf{B}$ in the $y-z$ plane, at an angle $\varphi$ with the probe axis:

$$
\begin{aligned}
\frac{d^{2} v_{x}}{d t^{2}} & +\Omega_{e}^{2}\left[1-l_{e}^{2} \frac{\partial^{2}}{\partial x^{2}} \frac{e \Phi}{k T_{e}}\right] v_{x} \\
& =\left(v_{y} \frac{\partial}{\partial x}-\Omega_{e} \cos \varphi\right) \frac{\partial}{\partial y} \frac{e \Phi}{m_{e}}, \\
\frac{d^{2} v_{y}}{d t^{2}} & +\Omega_{e}^{2}\left[\cos ^{2} \varphi-l_{e}^{2} \frac{\partial^{2}}{\partial y^{2}} \frac{e \Phi}{k T_{e}}\right] v_{y} \\
& =\left(v_{x} \frac{\partial}{\partial y}+\Omega_{e} \cos \varphi\right) \frac{\partial}{\partial x} \frac{e \Phi}{m_{e}}+\Omega_{e}^{2} v_{z} \sin \varphi \cos \varphi .
\end{aligned}
$$

Equations (33a), (33b) were obtained by deriving the respective equations of motion and using the derivative along the electron orbit, ${ }^{15}$

$$
\frac{d}{d t} \frac{\partial \Phi}{\partial x}=v_{x} \frac{\partial^{2} \Phi}{\partial x^{2}}+v_{y} \frac{\partial^{2} \Phi}{\partial x \partial y},
$$

and similarly for $d(\partial \Phi / \partial y) / d t$. Unless $B$ is small the motion is $\varphi$-dependent; it is rotationally symmetric for $\varphi=0$, and free of magnetic force in (33b) for $\varphi=\pi / 2$.

For large $B$, Eqs. (33a), (33b) would describe gyromotion, as represented by the first term in the bracket on the left-hand-side, plus a small drift due to the electric field. We now assume, on the contrary, that $B$ is sufficiently small $\left(l_{e}\right.$ large enough) to make such effects negligible. We first use the $B=0$ solution of Sec. III to determine how small the magnetic field must be for gyromotion to be indeed negligible throughout both the orbital motion from the two thin layers to the probe, and the large potential rise inside those layers (Fig. 3). In the broad region between the probe and thin layers, the left-hand-side of Eq. (33a) takes the form

$$
\begin{aligned}
\frac{d^{2} v_{x}}{d t^{2}} & +\Omega_{e}^{2}\left[1-\frac{l_{e}^{2}}{R^{2}} \frac{\sigma_{1} T_{i} / T_{e}}{g\left(u_{P}\right)} \frac{r_{1}^{2}}{r^{2}}\right. \\
& \left.\times\left(\frac{x^{2}-y^{2}}{r^{2}} \frac{d g}{d u}+\frac{x^{2}}{r^{2}} \frac{d^{2} g}{d u^{2}}\right)\right] v_{x},
\end{aligned}
$$

with $r_{2} \cong r_{1}$ and $R \leqslant r<r_{1}$. Gyromotion is negligible if $R^{2} g\left(u_{p}\right) / l_{e}^{2}$ is small; note that $g\left(u_{p}\right)$ is only logarithmically large. Similarly, within the thin layers $\left(r / r_{1}\right.$ very close to unity) we have $N_{e}-N_{i} \sim N_{\infty}$ and $r d^{2} \Phi / d r^{2} \gg-d \Phi / d r$, and the left-hand-side of (33a) becomes

$$
\frac{d^{2} v_{x}}{d t^{2}}+\Omega_{e}^{2}\left[1-\frac{l_{e}^{2}}{\lambda_{D e}^{2}} \frac{x^{2}}{r^{2}} \frac{N_{e}-N_{i}}{N_{\infty}}\right] v_{x} ;
$$

gyromotion is negligible here if $\left(\lambda_{D e} / l_{e}\right)^{2}$ is small.

For magnetic effects to be fully ignorable, the drift (second) term on the right-hand-side of Eqs. (33a), (33b) should be small too. This easily leads to conditions $R \sqrt{g\left(u_{p}\right)} \cos \varphi / l_{e} \ll 1$ and $\lambda_{D e} \cos \varphi / l_{e} \ll 1$ for the broad region and the thin layers, respectively. All this means, first, that, for $B$-effects to be negligible, the ratio $\lambda_{D e} / l_{e}$, in addition to the Parker-Murphy parameter $R / l_{e}$ in Eq. (32), must be small. Second, $B$-effects will be weaker for a magnetic field nearly perpendicular to the probe axis. Finally, $R / l_{e}$-effects will be weaker for $T_{e} / T_{i}$ small [see Eq. (34a)].

At the relatively high densities of the $F$-layer $\left(N_{\infty}\right.$ $\left.=10^{11}-10^{12} \mathrm{~m}^{-3}\right), \lambda_{D e} / l_{e} \propto B / \sqrt{N}_{\infty}$ is indeed small, but it reaches above unity at extreme altitudes. Experiments on board an elliptical-orbit satellite ${ }^{16}$ and a rocket, ${ }^{17}$ covering a broad range of altitudes, did show a current dependent on the angle between $\mathbf{B}$ and a cylindrical probe ( $B$-effects) when $N_{\infty}$ dropped low enough, at very low and high altitudes. In all such experiments, probe bias was only moderately high, however.

\section{CONCLUSIONS}

Bare tether applications are based on the assumption that the tether collects electrons in the OML regime of cylindrical Langmuir probes. The definite and simple OML current law, which allows for detailed design considerations, has opened the way to a technology of electrodynamic tethers. ${ }^{7-9}$ Here, we have determined the domain of OML validity in parameter space; we studied the surface bounding that domain as a relation among the dimensionless numbers,

$$
R / \lambda_{D e}, e \Phi_{p} / k T_{e}, T_{i} / T_{e}, \text { and } \lambda_{D e} / l_{e},
$$

for the very large $e \Phi_{p} / k T_{e}$ values of interest and different tether geometries.

We found that the ratio $\lambda_{D e} / l_{e}$ must be small for magnetic effects-which would break the OML law otherwise-to be ignorable. This ratio is a property of the plasma rather than a free design parameter. In the Earth's ionosphere $\lambda_{D e} / l_{e}$ is small for $N_{\infty}$ above $10^{11} \mathrm{~m}^{-3}$; this breaks down at low, and sufficiently high, altitudes. Magnetic effects are weaker for $\mathbf{B}$ near perpendicular to the probe axis. 
For $\lambda_{D e} / l_{e}$ small we determined the maximum radius for the OML regime to hold, giving

$$
R_{\max } / \lambda_{D e} \text { vs } e \Phi_{p} / k T_{e}, \text { and } T_{i} / T_{e} .
$$

$R_{\max }$ exhibits a minimum as a function of $\Phi_{p}$ but, at the bias of interest, is slowly increasing, and above $\lambda_{D e}$ in the ionospheric case $\left(T_{i} / T_{e} \sim 1\right)$. For $\lambda_{D e} / l_{e}$ small and $R \sim \lambda_{D e}$, we have $R / l_{e}$ small too, a second condition required for magnetic effects to be weak. We also found $R_{\max } / \lambda_{D e}$ increasing with $T_{i} / T_{e}$. If a thin tape is used instead of a wire (with all others parameters equal), the maximum valid width is found to be $4 R_{\max }$. Trapped-orbit effects should be negligible for bare tether conditions.

Important questions left out of this study are the possible effects of a relative motion of plasma and probe, how the current lags behind the OML current when the probe is too thick, and the interference between two probes at a finite distance. Work is in progress on these questions.

\section{ACKNOWLEDGMENTS}

We acknowledge fruitful comments from the referee.

The work of J. R. Sanmartín was supported by the Comisón Interministerial de Ciencia y Tecnolóia (Spain) under grant No. PB94-0417-C03-01. The work of R. D. Estes was partially funded by NASA Grant No. NAG8-1303.

\section{APPENDIX A: THIN TRANSITIONAL LAYERS}

Since $-d \Phi / d r$ (and $d^{2} \Phi / d r^{2}$ ) in the quasineutral solution below point 1 (Fig. 3) tends to infinity as $r \rightarrow r_{1}$, one must retain the left-hand-side of Eq. (1) in a narrow neighborhood of that point. Throughout this layer, we have $J_{r}^{*}(E)=J_{e n v}(E)$, and may thus use $N_{e} / N_{\infty}$ as given in (18). Expanding (1) around $r_{1}, \Phi_{1}$, one finds

$$
\lambda_{D i}^{2} \frac{d^{2}}{d r^{2}} \frac{e \Phi}{k T_{i}}=\mu \frac{r_{1}-r}{r_{1}}+\frac{\lambda}{2}\left(e \frac{\Phi-\Phi_{1}}{k T_{i}}\right)^{2},
$$

where $\mu\left(T_{e} / T_{i}\right)$ and $\lambda\left(T_{e} / T_{i}\right)$ are given by

$$
\begin{aligned}
\mu \equiv & \int_{0}^{\infty} \frac{d E \exp \left(-E / k T_{e}\right)}{\pi k T_{e}}\left[2 \sqrt{\frac{J_{\text {env }}^{2}(E)}{J_{r_{1}}^{2}(E)-J_{e n v}^{2}(E)}}\right. \\
& \left.-\sqrt{\frac{J_{r_{1}}^{2}(E)-J_{R}^{2}(0)}{J^{2}(0)}}\right], \\
\lambda \equiv & -\exp \left(-\frac{e \Phi_{1}}{k T_{i}}\right)+\int_{0}^{\infty} \frac{k T_{i}^{2} d E \exp \left(-E / k T_{e}\right)}{4 \pi T_{e}\left(E+e \Phi_{1}\right)^{2}} \\
& \times\left[2 J_{e n v}(E) \frac{3 J_{r_{1}}^{2}(E)-2 J_{e n v}^{2}(E)}{\left(J_{r_{1}}^{2}(E)-J_{e n v}^{2}(E)\right)^{3 / 2}}\right. \\
& \left.-J_{R}(0) \frac{3 J_{r_{1}}^{2}(E)-2 J_{R}^{2}(0)}{\left(J_{r_{1}}^{2}(E)-J_{R}^{2}(0)\right)^{3 / 2}}\right] .
\end{aligned}
$$

Defining

$$
\frac{r_{1}-r}{r_{1}} \equiv \beta \xi \equiv\left[\sqrt{\frac{2}{\mu \lambda}} \frac{\sigma_{1} k T_{i}}{e \Phi_{P}} \frac{\lambda_{D i}^{2}}{R^{2}}\right]^{2 / 5} \xi,
$$

$$
\frac{e \Phi-e \Phi_{1}}{k T_{i}} \equiv \sqrt{\frac{2 \mu \beta}{\lambda}} Y,
$$

Eq. (A1) becomes ${ }^{12}$

$$
d^{2} Y / d \xi^{2}=Y^{2}+\xi
$$

As $\xi \rightarrow-\infty$, the solution to (A5) must match smoothly the quasineutral solution near point $1,-Y \approx \sqrt{-\xi}$; this determines uniquely $Y(\xi)$, which is a parameter-free function. As $\xi \rightarrow \xi_{2} \approx 3.45, Y(\xi)$ diverges as $Y \approx 6 /\left(\xi-\xi_{2}\right)^{2}$, and Eq. (A3) fails locally. At $r \approx r_{2}$ there is a new thin layer where the left-hand-side of (1) keeps the form of (A1), but the full expressions (2) and (18) for $N_{i}$ and $N_{e}$ must be retained, except that $J_{r}^{2}$ in (18) simplifies to $J_{r}^{2}(E) \approx 2 m_{e} r_{2}^{2}(E$ $+e \Phi)$. At the top of this second layer, one finds $\Phi(r)$ $\propto\left(r_{2}-r\right)^{4 / 3}$

Setting $\xi=\xi_{2}$ in (A4a) we have $r_{2}=r_{1}\left(1-\beta \xi_{2}\right)$. Defining $\sigma_{2} \equiv e \Phi_{P} R^{2} / k T_{i} r_{2}^{2}$, we then have $\sigma_{2} \cong \sigma_{1}\left[1+2 \beta \xi_{2}\right]$, or finally,

$$
\begin{aligned}
& \sigma_{2}=\sigma_{1}\left[1+2 \xi_{2}\left(\frac{2 \sigma_{1}^{2}}{\lambda \mu}\right)^{1 / 5}\left(\frac{k T_{i}}{e \Phi_{P}}\right)^{2 / 5}\left(\frac{\lambda_{D i}}{R}\right)^{4 / 5}\right], \\
& \xi_{2} \approx 3.45 .
\end{aligned}
$$

\section{APPENDIX B: TRAPPED ORBIT EFFECTS}

Our collisionless analysis clearly requires an overall mean free path $\lambda_{\text {coll }}^{\infty}$ for electron scattering by neutrals and charged particles large compared with the characteristic length of the collection process, $r_{0}$. This condition, however, may not rule out a secular collisional effect on the current $I$. In computing $I$, only $E>0$ electrons moving along unbounded orbits were considered; collisions, no matter how unfrequent, could make that solution inconsistent because the potential $\Phi(r)$ allows for bounded orbits. ${ }^{11,18}$ As current is collected at a steady rate, electrons are knocked into those orbits at a much lower rate. Calling "trapped" the bounded orbits that do not hit the probe, and "nontrapped" those that hit it, we write $N_{e}=N_{e u}+N_{e t}+N_{e n}$, all three types of orbits, unbounded, trapped, and nontrapped, contributing to $N_{e}$; in Secs. II and III we had set $N_{e}=N_{e u}$. Actually, electrons knocked into nontrapped orbits are rapidly lost to the probe and may be ignored: a steady regime $\left(\widetilde{\nu} N_{e n} / r_{0} \sim\right.$ a fraction of $\left.\widetilde{\nu} N_{e} / \lambda_{\text {coll }}^{\infty}\right)$ sets up in times $\sim r_{0} / \widetilde{\nu}$, where $\widetilde{\nu}$ is a characteristic velocity, $N_{e n} / N_{e}$ thus vanishing with the ratio $r_{0} / \lambda_{\text {coll }}^{\infty}$.

The case for $N_{e t}$ is different. If there is no faster mechanism for orbital loss, $N_{\text {et }}$ growth would be finally limited by collisions knocking trapped electrons into unbounded or nontrapped orbits; the ratio $N_{e t} / N_{e u}$ would involve no small parameter, and might, in principle, have any value, and thus affect $\Phi(r)$ itself, and $I$. For probes of length $L \ll \lambda_{\text {coll }}^{\infty}$, however, an orbital-loss mechanism faster than collisions does exist: trapped electrons are unhindered to move along the probe axis, and thus escape through the probe ends. ${ }^{11}$ With a steady regime being established in times $\sim L / \widetilde{\nu}, N_{e t} / N_{e}$ would vanish with the ratio $L / \lambda_{\text {coll }}^{\infty}$, leaving $I$ unaffected. Actually, as we shall now see, the current in our problem 
remains unaffected under the weaker condition $L$ $\leqslant \lambda_{\text {coll }}^{\infty}($ Coulomb $) \ll \lambda_{\text {coll }}^{\infty}($ neutrals $)$, which is typically satisfied in the tether case.

The local condition for maximum angular momentum at given energy, $J^{2}<J_{r}^{2}(E)$, can be rewritten as a condition for minimum $E$ at given $J$,

$$
E>U_{J}(r) \equiv \frac{J^{2}}{J_{R}^{2}(0)} \frac{e \Phi_{P} R^{2}}{r^{2}}-e \Phi(r),
$$

with the first term of the effective potential energy $U_{J}(r)$ written conveniently. Bounded orbits lie around minima of $U_{J}(r)$. The equation locating $U_{J}(r)$-extrema at given $J$ is

$$
\frac{d \Phi}{d\left(\Phi_{P} R^{2} / r^{2}\right)}=\frac{J^{2}}{J_{R}^{2}(0)},
$$

where the left-hand-side is just the slope of the profile in Fig. 3 , drawn for the no- $N_{e t}$ case. This slope is small at the probe and increases with $r$, diverging at $r=r_{1}$; it then decreases to unity at $r_{0}$, with a further drop there (the full Poisson equation was needed at $r_{0}$, in Sec. III, to locally rotate the quasineutral profile in Fig. 3, making it tangent to the diagonal from below). The slope increases again beyond $r_{0}$, diverging as $r \rightarrow \infty$.

The entire behavior of $U_{J}(r)$ can be read off Fig. 3, the $J$-term in (B1) representing straight lines through the origin. For $J=J_{R}(0)$, i.e., for the diagonal, $U_{J}(r)$ vanishes at $r$ $=R, r=r_{0}$, and $r \rightarrow \infty$, and is negative elsewhere; there is a maximum at $r_{0}$, and minima at values $r<r_{1}$ and $r>r_{0}$ where the profile is parallel to the diagonal, producing inner and outer sets of $E<0$ trapped orbits. For $J>J_{R}(0), U_{J}(r)$ is positive at both probe and maximum, which lies between $r_{1}$ and $r_{0}$; there are now inner trapped orbits with $E>0$. For $J<J_{R}(0)$, the maximum stays at $r_{0}$, until finally disappearing with the approaching outer minimum.

Consider $N_{e t}$ for the range $r_{0} \leqslant r<\infty$, where all bounded orbits have $E<0$. In the hypothetical case of a nonabsorbing probe having $L / \lambda_{\text {coll }}^{\infty}$ large, $N_{e t}$ (here effectively identical to $\left.N_{e t}+N_{e n}\right)$ would be limited by collisions that establish thermal equilibrium in the bounded-orbit domain by knocking electrons back into unbounded orbits; Eq. (4), with $d J$ $\rightarrow 2 d J$ and both signs of $\nu_{r}$ counted, would give

$$
\begin{aligned}
N_{e t}=N_{e t}(e q) & \equiv \int_{-e \Phi}^{0} \frac{d E}{k T_{e}} \exp \left(\frac{-E}{k T_{e}}\right) \int_{0}^{J_{r}(E)} \frac{2}{\pi} \frac{d J}{\sqrt{J_{r}^{2}(E)-J^{2}}} \\
& =\exp \left[\frac{e \Phi(r)}{k T_{e}}\right]-1,
\end{aligned}
$$

while the second term in Eq. (9) for $N_{e u}$ (arising from current collection) would vanish, yielding the Boltzmann law for $N_{e}$. For our absorbing probe the full equation (9) naturally applies. Also, $N_{e t}$ is reduced below $N_{e t}(\mathrm{eq})$ because, first, nontrapped orbits in the bounded-orbit domain are unpopulated and, second, the population in the trapped subdomain does not reach equilibrium values, the steady electron flux from trapped to nontrapped orbits requiring a net flux from unbounded to trapped orbits. ${ }^{18}$ A value $L / \lambda_{\text {coll }}^{\infty} \leqslant 1$ further reduces $N_{e t}$ and, additionally, leads to a value $N_{e t} / N_{e u}$ $\leqslant 1$.
Crucial points now are (1) there is no trapped-orbit subdomain at $r_{0}$, and (2) $\Phi\left(r \geqslant r_{0}\right)$ is determined by a local relation (quasineutrality). Note that all $E<0$ electrons at $r_{0}$ have $J \leqslant J_{R}(0)$; for such $J$ there is no $U_{J}(r)$-maximum between $r_{0}$ and $R$, while, as seen in (B1), $U_{J}(R)$ is less than $U_{J}\left(r_{0}\right)$. All bounded orbits are thus nontrapped, a result giving $N_{e t}\left(r_{0}\right) \equiv 0$. With $N_{e u}\left(r_{0}\right)$ taken from (9), quasineutrality at $r_{0}$ still leads to Eqs. (14a), (14b). For the $N_{e t}\left(r>r_{0}\right)$ space-charge to invalidate (9), it had to substantially lower the profile in Fig. 3 beyond $r_{0}$, dropping locally below the diagonal. The ratio $N_{e t} / N_{e}$, however, vanishes for both $r$ $=r_{0}$ and $r \rightarrow \infty$, and will have a maximum, at some $r>r_{0}$, (moderately) small.

The results in Sec. III for both point 1 and the broad region from $r_{1}$ to $R$ (Fig. 3) remain valid too. Note that no $U_{J}(r)$ has a maximum in this range, and that electrons incoming along unbounded orbits become highly energetic past $r_{1}$, where the potential rises steeply (and rejects ions). With $\left(m_{e} / 2\right) \nu^{2} \approx e \Phi_{P} \gg k T_{e}$ near the probe and $N_{e} / N_{\infty} \ll 1$ closer to $r_{1}$, the characteristic mean free path for collisions that populate inner trapped-orbits satisfies, $\lambda_{\text {coll }}$ (electrons) $\gg \lambda_{\text {coll }}^{\infty}($ Coulomb $) \geqslant L$. This results in a small ratio $N_{e t} / N_{e}$ locally.

In the tether case, electrons escape trapped orbits both because of the finite collecting length $L$, and because $\Phi_{P}$, and the trapped-orbit estructure, vary along this length. Typical values are $L / 2 \sim \lambda_{\text {coll }}^{\infty}($ Coulomb $) \sim 1 \mathrm{~km} \ll \lambda_{\text {coll }}^{\infty}$ (neutrals). In the inner region, with $e \Phi_{P} \sim 100 \mathrm{eV}$, we would have $\lambda_{\text {coll }}$ (neutrals) $>30 \mathrm{~km}$ at altitudes exceeding $300 \mathrm{~km}$.

\section{APPENDIX C: POTENTIAL BARRIERS NEAR A THIN TAPE}

As seen in Sec. II, distribution function, transverse energy, and velocity $\nu_{z}$ are conserved along electron orbits independently of the conservation of angular momentum; they are thus conserved for the tape of Fig. 8 and, in general, for a cylinder of arbitrary cross section. At the probe, one would have $\quad 1 / 2 m_{e} \nu_{P \perp}^{2}-e \Phi_{P}=1 / 2 m_{e} \nu_{\infty \perp}^{2}, \quad \nu_{P z}=\nu_{\infty z}, \quad$ and $f\left(\bar{r}_{P}, \bar{\nu}_{P}\right)=f_{M}\left(\nu_{\infty}\right)$ if the $\bar{\nu}_{P \perp}\left(\nu_{P x}, \nu_{P y}\right)$ orbit, traced back from $\bar{r}_{P}$, reaches infinity. When this is the case for the full range $-\pi / 2<\psi_{P}<\pi / 2\left(\psi_{P} \equiv\right.$ angle between $\bar{\nu}_{P \perp}$ and inward normal) at each $\nu_{P \perp}$ and each point on the probe surface, the current $I$ comes out proportional to the probe area and independent of its shape, with the current density $j=I /(L$ $\times$ perimeter) uniform over the probe, ${ }^{13}$

$$
\begin{aligned}
j= & \frac{e N_{\infty} m_{e}}{2 \pi k T_{e}} \int_{-\pi / 2}^{\pi / 2} \cos \psi_{P} d \psi_{P} \int_{0}^{\infty} \nu_{\infty \perp} d \nu_{\infty \perp} \\
& \times \sqrt{\nu_{\infty \perp}^{2}+\frac{2 e \Phi_{P}}{m_{e}}} \exp \left(-\frac{m_{e} \nu_{\infty \perp}^{2}}{2 k T_{e}}\right) .
\end{aligned}
$$

Equation $(\mathrm{C} 1)$, where the $\nu_{P z}$-integration was already carried out and we changed variables from $\psi_{P}, \nu_{P \perp}$ to $\psi_{P}, \nu_{\infty \perp}$, recovers the OML law. For the wire of Sec. II, with all points on the probe surface equivalent, one finds $\left|\sin \psi_{P}\right| \approx J / J_{R}(0)$, recovering the old OML condition too: maximum $J$ $\left[\equiv J_{R}^{*}(E)\right]=J_{R}(E) \approx J_{R}(0) \rightarrow \operatorname{maximum}\left|\sin \psi_{P}\right|=1$. 
Attracted particles leaving (backward in time) a point of a probe not convex enough, might actually return to it at some other point, invalidating (C1). (For repelled particles, all orbits from a nonconcave probe reach back to infinity; this leads to the old result that current to a retarding probe is independent of probe shape. ${ }^{19}$ ) Such an orbit must become tangent from the inside to one of the equipotential lines, with a radius of curvature $\rho$ (orbit) $<\rho$ (equipot) at the turning point, where we have $m_{e} \nu_{\perp}^{2} / \rho$ (orbit) $=e|\nabla \Phi|$ and $1 / 2 m_{e} \nu_{\perp}^{2}$ $\approx e \Phi \gg k T_{e} \cdot{ }^{13}$ For the potential given by Eq. (29) we find

$$
\begin{aligned}
& \rho(\text { equipot })=a \frac{\left(\sin ^{2} \nu+\sinh ^{2} w\right)^{3 / 2}}{\sinh w \cosh w}, \\
& \rho \text { (orbit) }=2 a \frac{1-\alpha w}{\alpha} \sqrt{\sin ^{2} \nu+\sinh ^{2} w} .
\end{aligned}
$$

Since $1 / 2 \alpha \equiv\left[\ln \left(e \Phi_{P} / k T_{i} \sigma_{1}\right)-2 b\right]^{-1}$ is small, the condition $\rho$ (orbit) $<\rho$ (equipot) can only hold in a thin layer next to the tape $\left[0<w<1 / 2 \alpha \sin ^{2} \nu\right.$, or $0<y / a<1 / 2 \alpha\left(1-x^{2} / a^{2}\right)^{3 / 2}$, $|x|<a]$.

To determine how the potential barriers in this layer reduce the current, it will suffice to consider orbits leaving backward in time, from a point in the segment $0<x_{P i}<a$ of the tape, at a small, upward glancing angle to either right or left in Fig. 8. The $y$-equation of motion,

$$
m_{e} \nu_{x} \frac{d \nu_{y}}{d x}=e \frac{d \Phi}{d w} \frac{\partial w}{\partial y} \quad\left(\frac{d \Phi}{d w}=-\alpha \Phi_{P}\right),
$$

may be readily integrated by using small $\alpha, w, k T_{e} / e \Phi_{P}$, and $\nu_{y} / \nu_{x}$ approximations leading to $w \approx y / \sqrt{a^{2}-x^{2}}, \quad \nu_{x}$ $\approx \nu_{\perp} \approx \nu_{P \perp} \approx \sqrt{2 e \Phi_{P} / m_{e}}$. There is a glancing angle to the right $\gamma_{r}$ (left $\gamma_{l}$ ) for the orbit to return to the tape at $x$ $=a(x=-a)$. The current density $j$ in $(\mathrm{C} 1)$ is then reduced because the $\psi_{P}$-range of integration is (weakly) reduced:

$$
\int_{-\pi / 2}^{\pi / 2} \cos \psi_{P} d \psi_{P}=2 \rightarrow 2\left(1-\frac{\gamma_{r}^{2}+\gamma_{l}^{2}}{4}\right) .
$$

Both $\gamma_{r}$ and $\gamma_{l}$, and thus the reduction of $j$, are $x_{P i}$-dependent; we find $\gamma_{r}=\gamma_{l}=(\pi-2) \alpha / 4$ for $x_{P i}=0$, and $\gamma_{r}=0, \gamma_{l}=\pi \alpha / 4$ for $x_{P i}=a$. The overall current reduction is about $\alpha^{2} / 8$, or about one percent for the $e \Phi_{P} / k T_{e}$ values of interest.

${ }^{1}$ M. Martínez-Sanchez and D. E. Hastings, J. Astronaut. Sci. 35, 75 (1987).

${ }^{2}$ R. D. Estes, J. Geophys. Res. 93, 945 (1988); J. R. Sanmartin and M. Martinez-Sanchez, ibid. 100, 1677 (1995).

${ }^{3}$ J. R. Sanmartín and R. D. Estes, J. Geophys. Res. 102, 14625 (1997).

${ }^{4}$ M. Martínez-Sanchez and J. R. Sanmartín, J. Geophys. Res. 102, 27257 (1997).

${ }^{5}$ P. Wilbur and T. Laupa, Adv. Space Res. 8, 221 (1988).

${ }^{6}$ M. J. Gerver, D. E. Hastings, and M. Oberhardt, J. Spacecr. Rockets 27, 391 (1990).

${ }^{7}$ J. R. Sanmartín, M. Martínez-Sanchez, and E. Ahedo, J. Propul. Power 9, 353 (1993).

${ }^{8}$ R. D. Estes, E. Lorenzini, J. R. Sanmartín, J. Pelaez, M. MartínezSanchez, L. Johnson, and I. Vas, "Bare tethers for electrodynamic spacecraft propulsion,', submitted to J. Spacecr. Rockets.

${ }^{9}$ R. D. Estes, J. R. Sanmartin, and M. Martinez-Sanchez, "Technology of bare tether current collection,', submitted to J. Spacecr. Rockets.

${ }^{10}$ I. B. Bernstein and I. N. Rabinowitz, Phys. Fluids 2, 112 (1959).

${ }^{11}$ National Technical Information Service Document No. AD 634596 (J. G. Laframboise, "Theory of spherical and cylindrical Langmuir probes in a collisionless, Maxwellian plasma at rest,' University of Toronto Institute for Aerospace Studies Report No. 100, 1966). Copies may be ordered from the National Technical Information Service, Springfield, VA 22161.

${ }^{12}$ S. H. Lam, Phys. Fluids 8, 73 (1965).

${ }^{13}$ J. G. Laframboise and L. W. Parker, Phys. Fluids 16, 629 (1973).

${ }^{14}$ L. W. Parker and B. L. Murphy, J. Geophys. Res. 72, 1631 (1967).

${ }^{15}$ N. Singh and V. S. Chaganti, J. Geophys. Res. 99, 469 (1994).

${ }^{16}$ N. J. Miller, J. Geophys. Res. 77, 2851 (1972).

${ }^{17}$ E. P. Szuszczewicz and P. Z. Takacs, Phys. Fluids 22, 2424 (1979).

${ }^{18}$ Y. L. Alpert, A. V. Gurevich, and L. P. Pitaevskii, Space Physics with Artificial Satellites (Consultants Bureau, New York, 1965), Chaps. VIII and IX.

${ }^{19}$ Yu. M. Kagan and V. I. Perel, Sov. Phys. Usp. 6, 767 (1964). 